\title{
SECULAR PERSPECTIVE ACCELERATION
}

Peter van de Kamp

Sproul Observatory

The secular perspective acceleration in the proper motion of a star is directly proportional to the parallax $\underline{\mathrm{p}}$, proper motion $\mu$, and radial

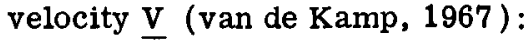

$$
\Delta \mu=-2 ! 05 \times 10^{-6} \underline{\mathrm{v}} \mu \underline{\mathrm{p}} / \text { year }
$$

The largest effect known is for Barnard's star, for which

$$
\begin{aligned}
& \underline{\mathrm{p}}=+0 ! 552 \pm ! 001 \quad(\text { p.e. }) \\
& \left.\mu_{\underline{x}}=-0 ! ! 8038 \pm ! 0001\right) \\
& \left.\mu_{\underline{y}}=+10 ! 2745 \pm .0001\right) \quad \text { total } \mu=10 ! 31 \text { in } 355: 5 \\
& \underline{\mathrm{V}}=-108 \pm 2.5 \mathrm{~km} / \mathrm{sec}
\end{aligned}
$$

The predicted perspective acceleration is:

$$
\begin{aligned}
& \left.\Delta \mu_{\hat{x}}=-0 ! \cdot 00010 \pm .00001 ;\right) \\
& \left.\Delta \mu_{\hat{y}}=+0 ! \cdot 00125 \pm .00003\right)
\end{aligned} \quad \text { total } \Delta \mu=+0 ! 00125 \pm 0 ! 00003
$$

Earlier provisional attempts to measure this acceleration were made by Lundmark and Luyten (1922), Alden (1924) and van de Kamp (1935). Sufficient observational material and time interval are now available to measure the quadratic time effect in the proper motion with high accuracy. The latest values, based on some 3500 plates taken with the Sproul refractor over the interval 1916-1919 and 1938-1969 yield the following acceleration components:

$$
\begin{aligned}
& q_{\hat{x}}=+! 000148 \pm 0 ! 000008 \\
& q_{\hat{y}}=+! 000528 \pm 0 ! 000008
\end{aligned}
$$

These values require a substantial correction for the spurious acceleration due to the changing influence of the proper motions of the reference stars as time goes by.

The proper motions of the reference stars were determined by referring them to a system of twenty-four background stars, using two pairs of plates with an interval of forty-four years. 
These resulting corrections for spurious acceleration are:

$$
\begin{aligned}
& +2\left[\underline{\Delta D}_{\underline{x}}\right]=-! 00026 \pm ! 00003 \\
& +2\left[\underline{D}_{\underline{y}}\right]=+.00067 \pm .0000 \Omega
\end{aligned}
$$

Here $\Delta \mathrm{D}$ are the annual changes in dependences of the reference stars with proper motion components $\mu_{x}, \mu_{y}$ (van de Kamp, 1967). Thus we find the following values for the true secular perspective acceleration:

$$
\begin{aligned}
& \left.\Delta \mu_{\mathrm{X}}=-0.90011 \pm 0.00003\right) \\
& \Delta \mu_{y}=+0.00120_{ \pm} 0.00003 \text { ) } \\
& \text { total } \Delta \mu=+0 ! 00121 \pm 0: 00003
\end{aligned}
$$

in excellent agreement with the predicted acceleration.

The small internal probable error for the observed yearly acceleration is not necessarily a measure for the true accuracy, which is affected by the perturbations (van de Kamp, 1969), and by possible systematic errors, particularly any introduced by the parallax series due to different emulsion and filter.

A significant source of error is the limited accuracy with which the spurious acceleration is determined. Improved values for the proper motions of the three reference stars are desired, an aim which ideally would require a background of galaxies but more realistically a larger time interval and/ or additional background stars besides those now available on the Sproul plates.

The principal source of error in the predicted value of the acceleration is any uncertainty in the observed value of the radial velocity. As a matter of fact we are now in a position to determine the radial velocity from the (true) secular perspective acceleration, parallax and proper motion being known with high accuracy. Using the total proper motion and acceleration, we thus find:

$$
\underline{\mathrm{V}}=-104 \pm 2.6 \mathrm{~km} / \mathrm{sec}
$$

While in the present case it may very well be possible to improve the value of the radial velocity from observations of the Doppler shift, the procedure just carried out has the value of deriving the radial velocity by a purely geometric method. This was pointed out long ago by Oort (1932) and others as a means of separating any gravitational red shift from Doppler shift. 


\section{References}

Alden, H. L. , 1924, Astron. J. 35, 133.

van de Kamp, P., 1935, Astron. J. 44, 73.

, 1935, Astron. J. 44, 74.

, 1967, Principles of Astrometry,

W. H. Freeman and Co. , $127 \mathrm{f}$.

, 1969, Astron. J. 74, 757.

Lundmark, K. , and Luyten, W. J. , 1922, Publ. Astr. Soc. Pac. 34, 126, 229. Oort, J. H. , 1932, Bull. Astr. Inst. Neth. 6, 287.

\section{DISCUSSION}

Dieckvoss: About twenty years ago, H. Werner at Zeiss, determined acceleration and proper motion of Groombridge 1830. Was it successful from absolute position?

van de Kamp: Yes, I believe some of these values are rather good. I don't have the figures in my hands right now. Although, if you have systematic errors, of course, the position may trail off. Eventually this is the sort of thing that can be done better by photographic astrometry. Perhaps we should take into account the Astrographic Catalogue wherever possible.

Eichhorn: It occurred to me that maybe one could turn the problem around. The parallax is something that is essentially an annual effect, that no matter how long you wait you can not improve its determination on the annual basis. If you can improve the determination of the parallax and from the acceleration of the proper motion - as you can determine the radial velocity essentially instantly. Would it not be feasible, say, to wait for 200 years, something like that, and get parallaxes from radial velocities of proper motions.

van de Kamp: I fully agree with you that parallax work is frustrating in a sense because you always get this limited swing of parallax of \pm 1 , you can not do anything about it. However, maybe 200 years is a long time to wait. Parallaxes are urgently needed and I daresay can be determined with adequate accuracy for a large number of stars by existing methods.

Luyten: If you want to wait 200 years, it is much cheaper, I think, to send a spaceship to Pluto, and lengthen the baseline for parallax determination. 